\title{
Resonance Structure of Dynamic Fractional Stark Ladders in Laser-Driven Biased Superlattices
}

\author{
Tomohiro Karasawa ${ }^{a}$, Nobuya Maeshima ${ }^{\mathrm{a}, \mathrm{b}}$, and \\ Ken-ichi Hino ${ }^{a, b, *}$ \\ ${ }^{a}$ Doctoral Program in Frontier Science, Graduate School of Pure and Applied \\ Sciences, University of Tsukuba, Tsukuba, Ibaraki 305-8573, Japan \\ ${ }^{\mathrm{b}}$ Center for Computational Sciences, University of Tsukuba, Tsukuba, Ibaraki \\ 305-8577, Japan
}

\begin{abstract}
The resonance structure of an electronic Floquet state in a dynamic fractional Stark ladder (DFSL) is examined based on the scattering theory applied to a dressed potential resulting from renormalization of a laser-electron interaction to an original potential. Here, DFSL is realized in laser-driven biased superlattices with a fractional matching ratio of a Bloch frequency to a laser frequency. It is revealed that in contrast to a conventional understanding, the DFSL resonance position and lifetime tend to redshift and shorten, respectively, with an increase in strength of the laser field, and further, these show irregular changes in a limited region of the strength. The underlying physics is discussed in detail.
\end{abstract}

Key words: A. Semiconductors, A. Quantum Wells, D. Optical properties PACS: 78.67.Pt

\section{Introduction}

Recent studies of intense excitation of semiconductors under external ac-fields have provided a variety of intriguing phenomena relevant to coherent dynamics and coherent control along with the development of high-power terahertz $(\mathrm{THz})$ light sources [1] . It is known that an appropriately controlled periodic

* Corresponding author. Tel/fax: +81-29-853-4994

Email address: hino@bk.tsukuba.ac.jp (Ken-ichi Hino). 
$\mathrm{THz}$ drive to biased semiconductor superlattices (SLs), which is termed as Wannier-Stark ladders (WSLs), causes characteristic phenomena of photonassisted tunneling (PAT) and dynamic localization (DL) $[2,3]$. The THz drive makes a localized WSL electron delocalized because of the PAT, while quantum transport and diffusion are brought to an almost complete standstill when the DL is realized. These phenomena are governed by an electric matching ratio, $\zeta=\Omega / \omega$, where $\Omega$ represents a Bloch frequency given by $\Omega=e F_{0} d / \hbar$ with $e, F_{0}, d$ and $\hbar$ the elementary electric charge, strength of a bias field, a SL period, and the Plank constant divided by $2 \pi$, respectively, and $\omega$ represents a frequency of an applied laser field.

When $\zeta$ equals a positive integer $n$, a WSL level supported by a tilted quantumwell (QW) potential at the $l_{0}$ th site is coupled with other levels at the nearestneighbor $\left(l_{0} \pm 1\right)$ th sites by a $n$-photon resonant transition, resulting in the PAT and the DL [3]. On the other hand, when $\zeta$ equals a rational number of the form $p / q$ with $p$ and $q$ the prime numbers, a coupling with WSL levels situated at the $q$ th-nearest-neighbor $\left(l_{0} \pm q\right)$ th sites is induced by a $p$-photon resonant transition. This type of dynamic WSL (DWSL) is called as dynamic fractional Stark ladder (DFSL), in which a parent band is split into a series of quasienergy subbands [4-7]. There are $q$ such subbands when the quasienergy is restricted to a Brillouin zone of length $\omega$. Due to exponential reduction of a hoping matrix element between two Wannier functions at the sites of $l_{0}$ and $\left(l_{0} \pm q\right)$ for $q \geq 2$, this subband quasienergy is given by

$$
E_{s, p}^{(q)} \approx \frac{s}{p} \Omega, \quad(s=0, \cdots, q-1),
$$

where this is reckoned from the miniband center of the original SLs. Because all DFSL states are spatially localized, miniband formation attributable to PAT is no longer discerned. In view of these features of DWSL, it is known that the $\zeta^{-1}-E$ diagram with $E$ as quasienergy shows self-similarity, where $\zeta$ is changed with $F_{0}$ kept constant; such a fractal structure is characteristic of DFSL. The DFSL is conventionally understood within the single-miniband (SMB) picture based on the tight-binding model, except for Ref. [7].

The purpose of the present paper is to examine a resonance structure of DFSL, namely, the change of resonance position and lifetime with respect to strength of a laser field $F_{a c}$. It should be noted that DFSL is already made unstable to a certain extent by dc-Zener tunneling (dc-ZT) due to an effect of $F_{0}$, by which a DFSL localized state collapses into continuum through a tilted SL potential barrier. This situation is, needless to say, beyond the criterion of validity of the above-mentioned SMB picture, because the dc-ZT couples WSL levels originating from different SL minibands. With regard to an effect of a laser field, photon sidebands of DFSL states pertaining to different SL minibands are likely coupled each other by ac-Zener tunneling (ac-ZT) $[8,9]$. Unlike PAT, this effect also makes DFSL more unstable with an increase in $F_{a c}$. 
Because such instability is closely related with continuum states, the concerned DFSL problem should be tackled in the framework of the scattering theory, rather than the tight-binding model, by means of which continuum states are difficult to incorporate into theory. To the best of our knowledge, the studies of the instability of DWSL against $F_{a c}$ are scarce. In Refs. [7] and [10], the scattering theory was developed to this problem along with discussion of the instability. In the former, the DFSL problem of a modeled optical SLs was dealt with based on the Floquet-Bloch method, and in the latter, DL of DWSL with $\zeta=n$ was exclusively focused based on the R-matrix Floquet theory.

In this study, the DFSL problem is also solved based on the scattering theory in the frequency region of $\omega>>1$, in which the high-frequency approximation (HFA) is ensured [11]. This model allows us to simplify complicated multichannel scattering (MCS) equations resulting from the Floquet theory to a single-channel scattering (SCS) equation governed just by a dressed WSL potential, termed a ponderomotive potential. Such a dressed-potential picture serves to provide an intuitive understanding of underlying physics. This was applied to the studies of intense laser-atom interactions [11], and an impurity state in low-dimensional semiconductors under an intense laser field [12-14].

The following intriguing results are obtained. A quasienergy position of a DFSL state and the associated lifetime vary in an irregular manner with an increase in $F_{a c}$, as far as $F_{a c}$ is not strong. As $F_{a c}$ becomes further greater, the quasienergy position redshifts and the lifetime is more reduced in a monotonic manner. These results appear incompatible with those obtained by the SMB model, in which both effects of dc- and ac-ZTs are neglected. Because the manifestation of the above-mentioned self-similar structure relies fully on the SMB approximation, the existence of this structure characteristic of DFSL would be doubtful in a strict sense.

This paper is organized as follows. Section 2 describes the theoretical framework for tackling the present DFSL problem by means of the scattering theory. Section 3 presents the results and discussion. Section 4 gives the conclusion. Atomic units (a.u.) are used throughout unless otherwise stated.

\section{Theoretical Framework}

The Hamiltonian of the DWSL concerned here is given by [10]

$$
H(z, t)=\left[p_{z}-\frac{1}{c} A(t)\right] \frac{1}{2 m(z)}\left[p_{z}-\frac{1}{c} A(t)\right]+V(z)+F_{0} z
$$

where $p_{z}, m(z)$, and $V(z)$ represent a momentum operator in the direction of crystal growth along the z-axis, an effective mass of electron, and a SL 
confining potential, respectively. Further, $A(t)$ is a vector potential at time $t$ for the applied electric field, $F(t)=-\dot{A}(t) / c$, with $c$ the speed of light. $F(t)$ consists of a static bias of $F_{0}$ and a monochromatic laser drive with strength $F_{a c}$ and frequency $\omega$; namely, $F(t)=F_{0}+F_{a c} \cos \omega t$. As is shown in detail in Ref. [10], applying the Kramers-Henneberger (KH) transformation [15] and the Floquet theorem to the Schrödinger equation relevant to the Hamiltonian $H(z, t)$, the following coupled equations are obtained:

$$
\sum_{\nu=-N_{p h}}^{N_{p h}}\left[\mathcal{H}_{\mu \nu}(z)+\mu \omega \delta_{\mu \nu}-E \delta_{\mu \nu}\right] \psi_{\nu \beta}(z)=0
$$

where a quasienergy and the maximum number of Floquet-expansion terms are expressed as $E$ and $N_{p h}$, respectively. Further, $\mathcal{H}_{\mu \nu}(z)$ represents a time average of $\mathcal{H}(z, t)$ over $T=2 \pi / \omega$, where

$$
\mathcal{H}(z, t)=p_{z}\left\{\frac{1}{m[z+a(t)]}\right\} p_{z}+V[z+a(t)]+F_{0} z+v(z, t) .
$$

Here, the time average of a function $X(z, t)$ is defined as

$$
X_{\mu \nu}(z)=\frac{1}{T} \int_{0}^{T} d t \exp [-i(\mu-\nu) \omega t] X(z, t)
$$

In Eq. (4), a laser-electron interaction is renormalized to the original mass and potential, $m(z)$ and $V(z)$, in terms of the $\mathrm{KH}$ transformation, resulting in the dressed mass and potential, $m[z+a(t)]$ and $V[z+a(t)]$, respectively. It is supposed that these become $m_{\infty}$ and $V_{\infty}$ in the asymptotic region of $z=z_{a s}<0$ with $\left|z_{a s}\right|>>\max (\alpha, 1)$. Further, $a(t)=\alpha \cos \omega t$, where $\alpha$ is termed the ponderomotive radius corresponding to the excursion amplitude of a classical electron traveling under a laser field; this is given by

$$
\alpha=\frac{F_{a c}}{m_{\infty} \omega^{2}}
$$

In addition, $v(z, t)$ in Eq. (4) represents the residual part that has just small effects to $\mathcal{H}(z, t)$; for an explicit expression of $v(z, t)$, see Ref. [10]. Equation (3) is considered as the MCS equations, where a channel can be defined as a photon index $\beta$ with $-N_{p h} \leq \beta \leq N_{p h} ; \psi_{\nu \beta}(z)$ represents the $\nu$ th component of the $\beta$ th solution. Actually, for any $E$, an open boundary condition are imposed on this component because of $F_{0} z_{a s}+V_{\infty}+\mu \omega \rightarrow-\infty$.

Below, we concern the high frequency region, namely, $\omega>>1$, ensuring the HFA. Thus, the MCS equations of Eq. (3) become decoupled into a set of SCS equations, because the off-diagonal terms of $\mathcal{H}_{\mu, \nu \neq \mu}(z)$ vanish [11]. Therefore, 
it suffices to solve just the following SCS equation:

$$
\left[p_{z}\left\{\frac{1}{m[z+a(t)]}\right\}_{00} p_{z}+U(z)+v_{00}(z)-E\right] \phi(z)=0,
$$

where $U(z) \equiv V_{00}(z)+F_{0} z$ and $\phi(z) \equiv \psi_{00}(z) ;\{1 / m\}_{00}, V_{00}$ and $v_{00}$ represent the time-averaged function following Eq. (5). Because the Hamiltonian of Eq. (7) becomes identical to $\mathcal{H}_{a s}(z) \equiv p_{z}^{2} / 2 m_{\infty}+F_{0} z+V_{\infty}$ in the asymptotic region, the scattering boundary condition of $\phi(z)$ is expressed as a linear combination of Airy functions, $\operatorname{Ai}(\xi)$ and $\operatorname{Bi}(\xi)$. By use of the asymptotic forms of these functions, $\phi(z)$ becomes of the form

$$
\phi(z) \approx C(E) z^{-1 / 4} \sin \left(\xi+\frac{\pi}{4}+\delta(E)\right),
$$

where $C(E)$ and $\delta(E)$ represent a normalization constant and a phaseshift, respectively, and $\xi=\left(2 m_{\infty} F_{0}\right)^{1 / 3}\left[z-\left(E-V_{\infty}\right) / F_{0}\right]$.

The excess density of state (DOS), $\rho^{(e x)}(E)$, and the associated time-delay, $\tau(E)$, are defined as

$$
\rho^{(e x)}(E)=\sum_{\mu=-N_{p h}}^{N_{p h}} \frac{d \delta(E+\mu \omega)}{d E} ; \tau(E)=\frac{\rho^{(e x)}(E)}{N_{o}},
$$

where $N_{0}$ represents the number of open channels included [16,17]; $N_{o}=$ $2 N_{p h}+1$ in the present case, because all channels are open. $\tau(E)$ shows a peak structure, and this value is identical to a lifetime of a resonance state of DFSL. The excess DOS is also expressed as $\rho^{(e x)}(E)=\rho(E)-\rho^{(a s)}(E)$, where $\rho(E)$ and $\rho^{(a s)}(E)$ represent the DOS of the concerned DFSL and that of a fieldfree asymptotic state corresponding to $\operatorname{Ai}(\xi)$, respectively. Because $\rho^{(a s)}(E)$ exhibits only structureless continuum, the resonance structure observed in $\rho^{(e x)}(E)$ is considered to be almost similar to that in $\rho(E)$.

\section{Results and Discussion}

The actual calculations are implemented for the SL of 35/7ML-GaAs/ $\mathrm{Ga}_{0.75}$ $\mathrm{Al}_{0.25} \mathrm{As}(1 \mathrm{ML}=2.83 \AA)$, where a SL lattice constant is $d=225$, height of the confining QW potential is $V_{b}=7.8 \times 10^{-3}(0.21 \mathrm{eV})$, and effective masses of electron in the well and barrier regions of the QW are $m_{w}=0.0665$ and $m_{b}=0.0772$, respectively. Unless otherwise specified, here the DFSLs with $\zeta$ $=1 / 3$ and $2 / 3$ are considered, in which the Bloch frequencies are set equal to $\Omega=2.67 \times 10^{-3}$ and $5.33 \times 10^{-3}$, respectively; these values correspond to $F_{0}=1.19 \times 10^{-5}(61.3 \mathrm{kV} / \mathrm{cm})$ and $2.37 \times 10^{-5}(122 \mathrm{kV} / \mathrm{cm})$, respectively. Thus, the laser frequency is kept to be $\omega=8.0 \times 10^{-3}$ common to both cases of 


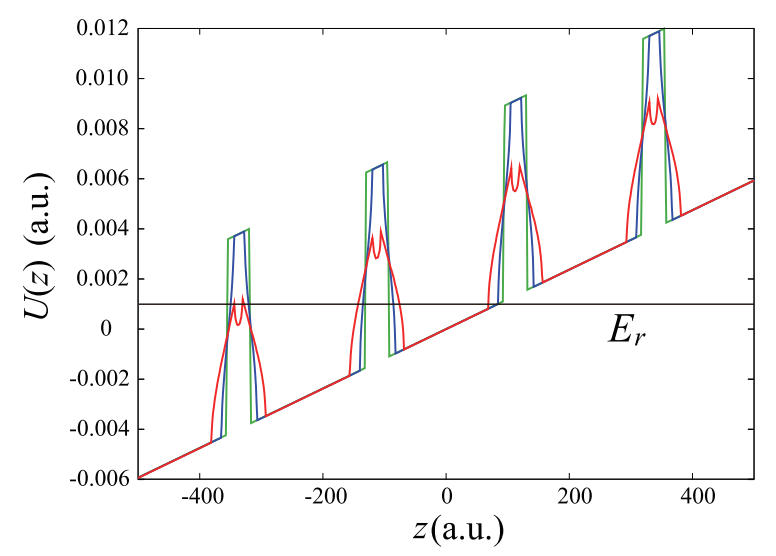

Fig. 1. Ponderomotive potential $U(z)$ as a function of $z$-coordinate for $\zeta=1 / 3$. Curves indicated by green, blue, and red solid lines represent potentials for $\alpha=1$, 10 , and 25, respectively. It is shown that the height of potential barrier decreases with an increase in $\alpha$. Solid line at $U(z)=E_{r}$ is depicted for the later use.

$\zeta$ 's. In practice, the validity of HFA is considered to be ensured if the condition of $\omega \gtrsim I_{p}$ is satisfied, where $I_{p}$ represents an ionization potential of a state of concern [11]. As is shown later, one mostly concerns the resonance state of the DFSL with quasienergy $E_{r} \approx 1.0 \times 10^{-3}$ attributed to the lowest SL miniband, and thus, $I_{p}$ is approximately equal to $5.5 \times 10^{-3}$ and $4.2 \times 10^{-3}$ for $\zeta=1 / 3$ and $2 / 3$, respectively, in view of potential drops due to $F_{0}$ of each case; that is, $I_{p} \approx V_{b}-\Omega / 2-E_{r}$ for small $\alpha$, and further, $I_{p}$ tends to be more reduced with an increase in $\alpha$. Therefore, it is verified that the above condition for the HFA is met in the present system. The SLs concerned are designed to be composed of ten QWs that are surrounded by $\mathrm{Ga}_{0.75} \mathrm{Al}_{0.25} \mathrm{As}$ in the outer regions. Hence, $m_{\infty}$ and $V_{\infty}$ are identical to $m_{b}$ and $V_{b}$, respectively.

Figure 1 shows the ponderomotive potential $U(z)$ for $\alpha=1,10$, and 25, corresponding to $F_{a c}=25.4,254$, and $635 \mathrm{kV} / \mathrm{cm}$, respectively, in the case of $\zeta=1 / 3 . U(z)$ is considered to be much dominant to another potential of $v_{00}(z)$ in Eq. (7) in the range of $\alpha$ discussed here. Hence, behavior of DFSL electrons is mostly determined by the ponderomotive potential under HFA; the mass variance incorporated in the first term of Eq. (7) would only minimally affect this behavior, because the difference between $m_{w}$ and $m_{b}$ is too small to cause a significant effect on DFSL. It is seen in Fig. 1 that with an increase in $\alpha$, the barrier height of $U(z)$ decreases accompanying a dip formation in the middle of the barrier region, and further, the barrier width tapers off; that is, the higher-energy and lower-energy portions of the barrier narrows and broadens, respectively, compared with the width of the original WSL potential. It should be noted that the alternative multiple-ionization mechanism termed as avalanche ionization (AI) [18] might become more important than DFSL at $F_{a c}=635 \mathrm{kV} / \mathrm{cm}$ corresponding to $\alpha=25$. However, calculations at this laser strength is just for the purpose of exploring the behavior of DFSL electron in such a high field region, rather than for the comparison with experiments. 

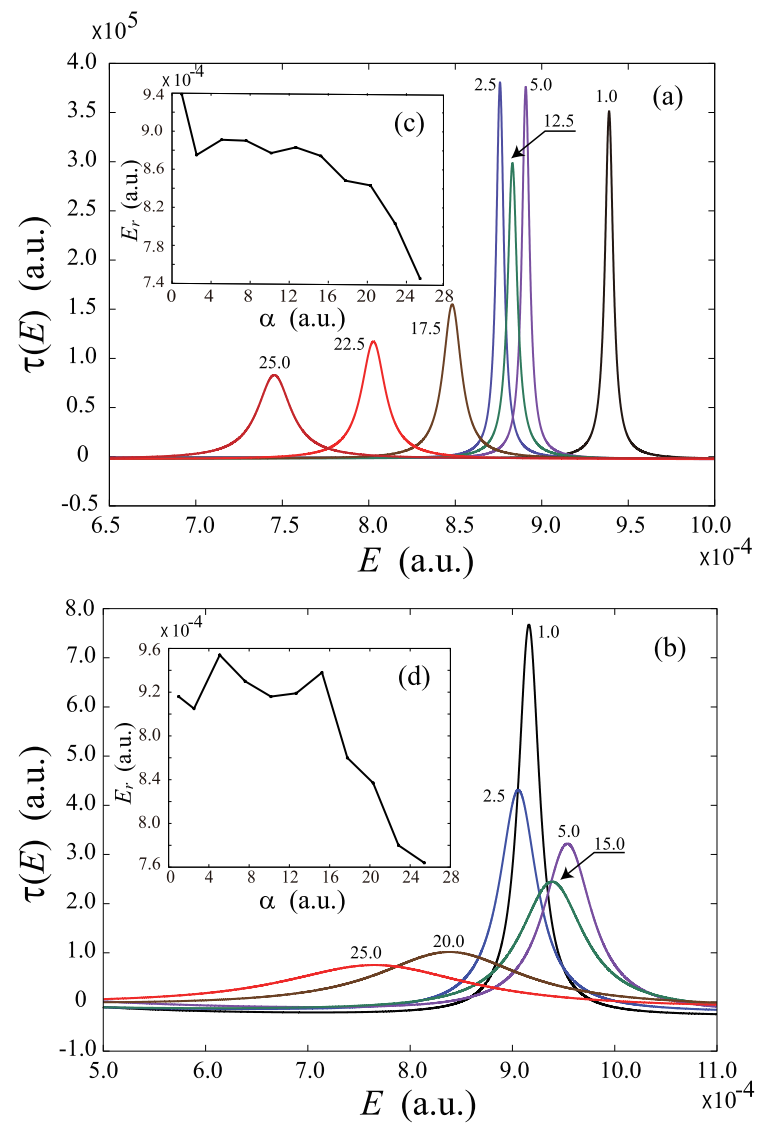

Fig. 2. Time-delay $\tau(E)$ as a function of quasienergy $E$ for $\zeta=1 / 3$ [panel (a)] and $2 / 3$ [panel (b)]. Figure attached around the top of every peak represents the value of $\alpha$. Inset: Peak position $E_{r}$ as a function of $\alpha$ for $\zeta=1 / 3$ [panel (c)] and 2/3 [panel (d)]. Calculated data for $\alpha=1,2.5,5,7.5,10,12.5,15,17.5,20,22.5$, and 25 are plotted, and connected by solid lines to aid the presentation.

Figures 2 (a) and 2 (b) show time-delay spectra $\tau(E)$ as a function of $E$ for $\zeta=1 / 3$ and $2 / 3$, respectively, in the range of $\alpha$ from 1 to 25 . The peak value of the spectra for $\alpha=1$ and its position are almost the same as the lifetime and the resonance energy, respectively, of a WSL state pertaining to the lowest SL miniband with a WSL index equal to zero. It is seen that the peak position $E_{r}$ of a DFSL resonance state varies in an oscillating manner for relatively small $\alpha$ 's, whereas this redshifts with a further increase in $\alpha$. Such irregularity is also shown in Figs. 2 (c) and 2 (d).

The variance of $E_{r}$ can be understood from the change of $U(z)$ with respect to $\alpha$. As $\alpha$ increases, the barrier height of $V_{00}(z)$, indicated by $h(\alpha)$, tends to decrease, while the width at the bottom of its potential barrier, indicated by $w(\alpha)$, tends to increase in general, as mentioned above. Therefore, in view of such a basic alteration of $U(z)$, its profile is replaced by a simple modeled potential, $\bar{U}(z)$, composed of a squared-well potential and a bias potential of $F_{0} z$. In this squared-well potential, height and width of the barrier are set to 


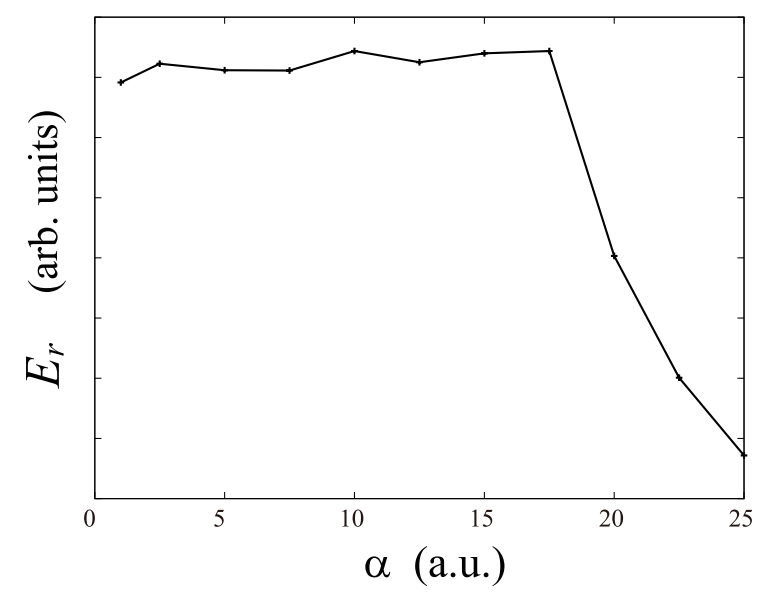

Fig. 3. The same as Fig. 2 (c) but the use of modeled potential $\bar{U}(z)$.

be equal to $h(\alpha)$ and $w(\alpha)$, respectively. Such a relatively simple change of $\bar{U}(z)$ with respect to $\alpha$ is attributed to the renormalization of a laser-electron interaction to $V(z)$. Figure 3 shows the change of $E_{r}$ obtained by the calculations using $\bar{U}(z)$ in place of $U(z)$. This result is considered to qualitatively well reproduce the irregularity seen in Fig. 2. Therefore, it would be concluded that the apparently complicated pattern of change in $E_{r}$ results from the relatively simple change of profile of $U(z)$. In conjunction with the change of $E_{r}$, it is seen that a binding energy of DFSL state decreases with an increase in $\alpha$. This is because $h(\alpha)$ is reduced by the order of $2 \times 10^{-3}$ when $\alpha$ changes from 1 to 25 , as shown in Fig. 1, whereas $E_{r}$ varies just by the order of $2 \times 10^{-4}$, as shown in Fig. 2. This is compatible with the discussion in Refs. [11].

With regard to the lifetime of DFSL state, this decreases overall in a monotonic manner with an increase in $\alpha$, as seen in Figs. 2 (a) and 2 (b). The pattern of change can also be understood from the change of $U(z)$ with respect to $\alpha$, though this would be seen intuitively just from the fact that the ac-ZT likely causes instability. In Fig. 1 , the solid line at $U(z)=E_{r} \approx 1.0 \times 10^{-3}$ is depicted to indicate that a resonance state with its peak position $E_{r}$ is dominantly supported by the QW situated in the center of the SLs, namely, in the region of $-90 \lesssim z \lesssim 90$. This line intersects the barrier region of $U(z)$ around $z=-113$ and -338 . With an increase in $\alpha$, the barrier width at the former intersection tends to broaden, whereas that at the latter one tends to narrow. An electron supported by the QW in the center of the SLs simultaneously feels the barrier-height reduction and the barrier-width broadening, both of which affect electron tunneling across the barrier around $z=-113$ in mutually incompatible manners; the tunneling is furthered by the former effect, however, suppressed by the latter one. For greater $\alpha$, the former effect is considered to be dominant to the latter one, and moreover, the barrier width at $z=-338$ becomes still narrower than that at $z=-113$ because of a potential drop by $\Omega$. Such changes of $U(z)$ lead to larger tunneling probabilities with the increase of $\alpha$, as seen in Fig. 2(a) and 2 (b). It should be noted that there is 
an exceptional case observed in the pattern of change for $\zeta=1 / 3$ in the small interval of $\alpha$ from 1 to 5 [see Fig. 2 (a)], where the lifetime varies slightly in an irregular manner. Such irregularity is considered to be attributed to the above two effects of $U(z)$ with comparable degree of magnitude.

In contrast to the results obtained here, the conventional SMB model leads to the result that a FDSL quasienergy position remains almost unchanged with respect to $F_{a c}$; the lifetime cannot be evaluated by this model. According to this, it is seen that in the $\zeta^{-1}-E$ diagram for the self-similar structure mentioned in Sec. 1 , some of quasienergies in a set of $E_{s, p}^{(q)}$ 's for a certain $p$ are identical to some of quasienergies in another set for a different $p$, where $q$ and $\Omega$ are given as constant. That is, following Eq. (1), there exists a combination of integers, $s$ and $s^{\prime}$, which satisfies the equality of $(s / p) \Omega=\left(s^{\prime} / p^{\prime}\right) \Omega$, where $s, s^{\prime}=0, \cdots,(q-1)$. For instance, in the case of $q=3$, we have $E_{0,1}^{(3)}=E_{0,2}^{(3)}$ and $E_{1,1}^{(3)}=E_{2,2}^{(3)}$. Here, $\omega$ for $p=1$ is half of that for $p=2$, and thus, $\alpha$ for $p=1$ is quadruple of that for $p=2$. Supposing that this relation is applicable to the case of Fig. 2 (c), the peak position for $\alpha=2.5$ and 5 would be required to be identical with that for $\alpha=10$ and 20, respectively. As seen in this figure, comparing $E_{r}$ 's of the pair of $\alpha=2.5$ and 10, the equality would prove somewhat true, whereas, comparing $E_{r}$ 's of the other pair of $\alpha=5$ and 20 , this is no longer correct. The same result is correctly applied to the case of Fig. 2 (d). To be summarized, the manifestation of the self-similar structure, which is characteristic of DFSL, is considered more unsure with an increase in $\alpha$. This is exclusively due to the effect of deformation of $U(z)$ attributable to ac-ZT that is absent from the SMB model.

\section{Conclusion}

Both of the redshift of $E_{r}$ and the reduction of $\tau\left(E_{r}\right)$ are revealed based on the SCS theory applied to the ponderomotive potential. Since this potential incorporates the effect of ac-ZT, the obtained result is attributable to a miniband coupling due to this effect. The change of $E_{r}$ is estimated to be $2 \sim 3 \mathrm{meV}$, and thus, this finding is expected to be confirmed by experiment. Because this result is obviously different from that obtained by the SMB model, the manifestation of the characteristic self-similar structure is considered doubtful in general; this feature appears to be justified just in a limited weak field region where the SMB picture still holds correctly. For irradiation of $\mathrm{THz}$ waves with smaller frequency where HFA is no longer valid, the present results would be modified to some extent, in which the MCS theory should be taken into account [10]. In addition, it is commented that according to the recent experiment of the THz-driven multiple QWs [19], an excitonic resonance position redshifts and the associated absorption linewidth broadens because of 
the dynamic Franz-Keldysh effect similar to the effect of the present DWSL.

\section{Acknowledgements}

This work was supported by a Grant-in-Aid for Scientific Research on Innovative Areas "Optical science of dynamically correlated electrons (DYCE)" (No. 21104504) of the Ministry of Education, Culture, Sports, Science and Technology (MEXT), Japan.

\section{References}

[1] S. D. Ganichev and W. Prettl, Intense Tetahertz Excitation of Semiconductors, (Oxford University Press, 2006).

[2] M. Holthaus, Phys. Rev. Lett. 69 (1992) 351.

[3] J. Zak, Phys. Rev. Lett. 71 (1993) 2623.

[4] X.-G. Zhao, R. J. Jahnke, and Q. Niu, Phys. Lett. A 202 (1995) 297.

[5] K. -C. Je, S. -H. Park, and Y. Kim, Phys. Rev. B 64 (2001) 075111.

[6] K. Yashima, K. Hino, and N. Toshima, Phys. Rev. B 68 (2003) 235325.

[7] M. Glück, A. R. Kolovsky, and H. J. Korsch, Phys. Rep. 366 (2002) 103.

[8] M. Holthaus and D. W. Hone, Philosophical Magazine B 74 (1996) 105.

[9] K. Hino, K. Yashima, and N. Toshima, Phys. Rev. B 71 (2005) 115325.

[10] A. Kukuu, T. Amano, T. Karasawa, N. Maeshima, and K. Hino, Phys. Rev. B 82 (2010) 115315.

[11] M. Gavrila, in Atoms in Intense Laser Fields ed. by M. Gavrila (Academic Press, New York, 1992) p. 435, and references cited therein.

[12] H. Sari, E. Kasapoglu, I. Sökmen, and N. Balkan, Semicond. Sci. Technol. 18 (2003) 470.

[13] Q. Fanyao, A. L. A. Fanyao, and O. A. C. Nunes, Phys. Rev. B 54 (1996) 16405.

[14] Y. P. Varshini, Superlatt. Microstruct. 30 (2001) 46.

[15] W. C. Henneberger, Phys. Rev. Lett. 21 (1968) 838.

[16] F. T. Smith, Phys. Rev. 118 (1960) 349.

[17] J. L. Kinsey, Chem. Phys. Lett. 8 (1971) 349.

[18] G. Juska and K. Arlauskas, Phys. Stat. Sol. (a) 59 (1980) 389.

[19] H. Hirori, M. Nagai, and K. Tanaka, Phys. Rev. B 81 (2010) 081305(R). 